\title{
ESTUDO COMPARATIVO DA EFICIÊNCIA DA DIFENILCARBAZIDA E DO ÓLEO DE COCO SAPONIFICADO MICROEMULSIONADOS NA INIBIÇÃO DA CORROSÃO DE AÇO CARBONO
}

\author{
Cátia G. F. T. Rossi, Hélio Scatena Jr. e Maria Aparecida M. Maciel* \\ Departamento de Química, Universidade Federal do Rio Grande do Norte, 59078-970 Natal - RN, Brasil \\ Tereza N. C. Dantas \\ Faculdade Natalense para o Desenvolvimento do Rio Grande do Norte/Departamento de Química, Universidade Federal do Rio \\ Grande do Norte, 59078-970 Natal - RN, Brasil
}

Recebido em 9/6/06; aceito em 19/12/06; publicado na web em 24/7/07

\begin{abstract}
COMPARATIVE EFFECTIVENESS MICROEMULSIONS OF DIPHENYLCARBAZIDE AND SAPONIFIED COCONUT OIL IN THE CARBON STEEL CORROSION INHIBITION PROCESS. The effectiveness of microemulsions (ME) of saponified coconut oil (OCS-ME) and diphenylcarbazide (DC-ME) on a carbon steel corrosion inhibition process was evaluated using an electrochemical method of polarization resistance. The ME was prepared with OCS, butanol, kerosene and saline solutions. OCS-ME and DC-ME showed highest inhibitions effects ( $77 \%$ and $92 \%$, respectively) at lower concentrations $(0.5 \%$ and $0.48-0.50 \%$, respectively). The surfactant OCS (in $\mathrm{H}_{2} \mathrm{O}$ ) showed lower efficiency ( $63 \%$ at $0.20-0.25 \%$ concentration). The greatest inhibitory effect of DC-ME could be correlated with the chemical structure and the rich O/W ME system, which are very important for adsorption phenomena in interfacial ME systems.
\end{abstract}

Keywords: diphenylcarbazide; saponified coconut oil; microemulsion.

\section{INTRODUÇÃO}

A importância do emprego de materiais metálicos em equipamentos utilizados no setor industrial (têxtil, de alimentos, de petróleo, dentre outros) que operam em altas temperaturas, justifica a necessidade da otimização de estudos sobre o processo de corrosão, objetivando a minimização de perdas por deterioração de metais. De maneira geral, o estudo de processos corrosivos tem como objetivo principal conhecer e caracterizar os diversos meios agressivos que são responsáveis pelas reações químicas e eletroquímicas causadas em materiais de elevada importância industrial para, em uma etapa subseqüente, reduzir os efeitos com agentes antioxidantes, denominados de inibidores de corrosão.

Os processos reacionais que geram a corrosão são geralmente espontâneos e transformam, constantemente, as características físicas e químicas de materiais metálicos e não-metálicos, interferindo, conseqüentemente, em sua estrutura, durabilidade e desempenho. $\mathrm{O}$ conhecimento exato dos princípios básicos desses processos é imprescindível para que se estabeleçam considerações qualitativas e quantitativas sobre a cinética de corrosão ${ }^{1}$. Dentre as muitas maneiras de definição de corrosão, pode-se dizer que "a corrosão é a deterioração espontânea de um material, metálico ou não metálico, por ação química ou eletroquímica do meio ambiente, aliada, ou não, a esforços mecânicos" ${ }^{\prime 1,2}$.

Um dos principais meios causadores de corrosão em instalações industriais (originárias de ferro ou aço) são as salmouras. Um exemplo significativo pode ser dado na indústria de óleos, onde a corrosão em oleodutos é desencadeada por elevados teores de salinidade, necessários para a produção de óleos ${ }^{3}$. Muitas pesquisas têm sido desenvolvidas no sentido de reduzir os efeitos da corrosão. Dentre os processos e métodos mais utilizados destacam-se: o aumento da resistência dos materiais por implantação de sistemas de proteção catódica e anódica, o uso de revestimentos, de agentes inibidores e antioxidantes ${ }^{1,4}$. O uso de inibidores de corrosão caracteriza-se em

\footnotetext{
*e-mail: mammaciel@hotmail.com
}

um dos métodos de grande interesse, já que funcionam como películas protetoras (sobre áreas anódicas e catódicas) que interferem na ação eletroquímica ${ }^{1}$. Como inibidores de corrosão, moléculas orgânicas fortemente polares são alvo de interesse na indústria de petróleo, já que promovem a formação de um filme protetor na interface metal-meio corrosivo ${ }^{5-8}$. Na indústria de óleos, por exemplo, destaca-se a classe dos tensoativos que, ao serem injetados sobre o óleo, promovem a formação de um filme superficial contendo grupos alquilorientados, que deslocam as moléculas de água (salina) da superfície metálica ${ }^{3}$. Um outro exemplo, consiste no uso de tensoativos aminados, que são injetados com fluidos de perfuração em poços de petróleo, para evitar a deterioração de hastes, bombas e tubulações de aço ${ }^{1,4}$. A classe das carbazidas consiste também, em bons inibidores de corrosão na indústria petrolífera, podendo-se destacar a difenilcarbazida (DC) $)^{9-12}$, bem como tiossemicarbazidas e seus deri$\operatorname{vados}^{13,14}$. Especificamente, a eficiência de uma série homóloga de tiossemicarbazidas (que diferem apenas no número de heteroátomos) aumentou com o número de heteroátomos presentes na molécula (usualmente obedece a seguinte ordem de inibição $\mathrm{P}>\mathrm{Se}>\mathrm{S}>\mathrm{N}>\mathrm{O})^{14}$. Comparativamente, a DC (Figura 1) é rica em grupos amina e possui ainda grupo carbonila e grupamentos fenila, disponibilizando, portanto, um grande número de elétrons em circulação.

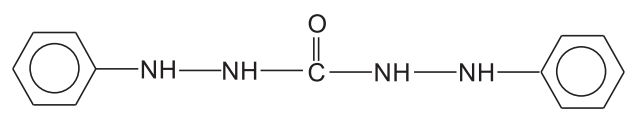

Figura 1. Estrutura química da DC

Geralmente, o uso de tensoativos como inibidores de corrosão se encontra bem documentado ${ }^{1,5,6,8,15-21}$. No entanto, tensoativos em sistemas microemulsionados (SME) como agentes inibidores de corrosão, bem como substâncias orgânicas solubilizadas em SME, são alvo recente de interesse do Laboratório de Tecnologia de Tensoativos da UFRN (LTT) ${ }^{22}$, não tendo sido evidenciado na literatura nenhum outro trabalho envolvendo SME como inibidores de corrosão. Portanto, a utilização de SME como meio inibidor de corrosão torna-se 
uma ferramenta eficaz já que promove maior solubilização de vários tipos de tensoativos (iônicos e não-iônicos) ou de moléculas orgânicas, ampliando seu poder anticorrosivo, que pode ser justificado pela ocorrência da adsorção do tensoativo na interface líquido-sólido formando uma camada protetora mais homogênea sobre o metal, o que possibilita maior contato interfacial devido à área apresentada pelas microestruturas formadas ${ }^{22}$. Esta alternativa torna-se viável uma vez que as microemulsões (ME) são sistemas que apresentam elevada estabilidade termodinâmica, são dispersos, coloidais translúcidos, monofásicos e opticamente isotrópicos ${ }^{22-29}$. Especificamente, as ME se formam a partir de uma aparente solubilização espontânea de dois líquidos imiscíveis (água, óleo) na presença de um tensoativo e, se necessário, um cotensoativo ${ }^{22-29}$. Atualmente, os estudos relacionados ao uso de tensoativos microemulsionados na inibição de processos corrosivos em superfície metálica mostraram-se promissores ${ }^{22}$. $\mathrm{O}$ fato de existirem em número muito reduzido, motivou a realização deste trabalho de investigação do poder anticorrosivo do tensoativo óleo de coco saponificado (OCS) solubilizado em água, bem como do OCS microemulsionado (OCS-ME) e da DC (Figura 1) em um sistema microemulsionado (DC-ME), onde o OCS é o tensoativo. Para tanto, a eficácia de OCS e dos sistemas microemulsionados (OCS-ME e DC-ME) foi avaliada em aço-carbono em contato com meio corrosivo, utilizando-se o método eletroquímico de resistência de polarização.

\section{PARTE EXPERIMENTAL}

\section{Análises físico-químicas do óleo vegetal}

O óleo de coco testado teve os seus índices oleoquímicos determinados de acordo com metodologia convencional ${ }^{30}$.

\section{Obtenção do óleo de coco saponificado (OCS)}

O óleo de coco foi saponificado pela diluição de 21,5 g de $\mathrm{NaOH}$ em $80 \mathrm{~mL}$ de água destilada; na seqüência, esta solução foi adicionada ao óleo de coco (100 g), seguido de adição de álcool etílico $(300 \mathrm{~mL})$. Esta mistura reacional foi aquecida em refluxo por $2 \mathrm{~h}$. Após remoção do álcool, o sabão obtido $(100 \mathrm{~g})$ foi seco a $40{ }^{\circ} \mathrm{C}$.

\section{Obtenção do sistema microemulsionado ${ }^{22,23}$}

Utilizando uma razão cotensoativo/tensoativo $\mathrm{C} / \mathrm{T}=1(30 \%)$, onde o OCS foi o tensoativo utilizado (15\%), o butanol $(15 \%$, cotensoativo), o querosene ( $10 \%$, fase oleosa, $\mathrm{F}_{\mathrm{o}}$ ) e a solução de $\mathrm{NaCl}$ [fase aquosa $\left(\mathrm{F}_{\mathrm{A}}\right)$, nas seguintes concentrações: 0,5, 1,5 e 2,5\%], obteve-se o sistema ME (base de cálculo $2 \mathrm{~g}$ ) com região de trabalho Winsor IV. Para tanto, titulou-se a matéria ativa (razão C/T) com a solução de $\mathrm{NaCl}\left(\mathrm{F}_{\mathrm{A}}\right)$, até atingir o ponto $\mathrm{S}$ de viragem (ponto de solubilização), caracterizado por uma mudança no aspecto físico do sistema, passando de turvo para límpido, ou vice-versa. Em seguida, obté-se um ponto de titulação (denominado de T) de composição conhecida dos constituintes da mistura, dentro da região monofásica do sistema pseudoternário. $\mathrm{O}$ ponto $\mathrm{T}$ é considerado o titulante dos binários: $\mathrm{F}_{\mathrm{A}}+\mathrm{F}_{\mathrm{O}}$ e $\mathrm{C} / \mathrm{T}+\mathrm{F}_{\mathrm{O}}$. A partir de um balanço de massas foram determinados os pontos limites das curvas de solubilidade das quatro regiões de Winsor (I, II, III e IV), no SME.

\section{Obtenção do diagrama de fase $^{22}$}

O procedimento utilizado para se obter a região de microemulsão (região IV) nos diagramas pseudoternários baseou-se no método que envolve a determinação dos pontos de solubilidades máximas da matéria ativa $\mathrm{C} / \mathrm{T}$ nas fases $\mathrm{F}_{\mathrm{A}}$ e $\mathrm{F}_{\mathrm{O}}$, por meio de titulações mássicas.

Obtenção de soluções, microemulsões e das concentrações dos inibidores de corrosão testados

Solução de OCS

Preparou-se uma solução padrão de OCS a $0,5 \%$, onde foram utilizados 1,21 g de OCS e $242 \mathrm{~mL}$ de água bidestilada.

Solução de $\mathrm{NaCl}$

Preparou-se uma solução padrão de $\mathrm{NaCl}$ a $0,5 \%$, onde foram utilizados $1,34 \mathrm{~g}$ de $\mathrm{NaCl}$ e $242 \mathrm{~mL}$ de água bidestilada.

\section{Sistema microemulsionado OCS-ME}

Realizou-se estudos do tensoativo OCS no SME em meio salino $(60 \%$ da solução de $\mathrm{NaCl} 0,5 \%)$. A Figura 2 ilustra no diagrama pseudoternário as três regiões de Winsor (WIV+S, WIV e WII) que foram obtidas.

Sistema microemulsionado DC-ME

$1,21 \mathrm{~g}$ de difenilcarbazida $(0,5 \%$ de $\mathrm{DC})$ correspondente ao gasto total necessário para as análises de eficiência de inibição a corrosão, foram solubilizados em $242 \mathrm{~mL}$ do sistema microemulsionado OCS-ME, para tanto, foi escolhido um ponto [0,5\% de solução salina $\left.(\mathrm{NaCl}) ; 30 \% \mathrm{C} / \mathrm{T} ; 60 \% \mathrm{~F}_{\mathrm{A}} ; 10 \% \mathrm{~F}_{\mathrm{O}}\right)$ ] na região Winsor WIV que caracteriza este sistema como sendo rico em água (O/A).

\section{Concentrações dos inibidores de corrosão testados}

As concentrações do OCS solubilizado em água, OCS microemulsionado (OCS-ME) e da difenilcarbazida solubilizada no sistema microemulsionado OCS-ME (denominada de DC-ME) foram obtidas de acordo com as variações dos volumes das soluções de $\mathrm{NaCl}$ 0,5\% (44 - $6 \mathrm{~mL}$ ), da massa do óleo de coco saponificado [OCS, 1,05 g (utilizada para a obtenção do sistema OCS-ME)] e do volume de OCS-ME 0,5\% (6 - $44 \mathrm{~mL})$. A Tabela 1 mostra estas variações, bem como os percentuais das concentrações dos sistemas inibidores OCS-ME e DC-ME (tendo variado entre 0,0681-0,5 e 0,0113-0,5\%, respectivamente) em função dos volumes destas soluções ( $\mathrm{NaCl}$ 0,5\% e OCS-ME 0,5\%).

Tabela 1. Percentual das concentrações dos sistemas OCS-ME e DC-ME em função dos volumes das soluções $\mathrm{NaCl}(0,5 \%)$ e OCS$\operatorname{ME}(0,5 \%)$

\begin{tabular}{lccc}
\hline $\mathrm{V}_{\mathrm{NaCl}}(\mathrm{mL})$ & $\mathrm{V}_{\mathrm{OCS}-\mathrm{ME}}(\mathrm{mL})$ & {$[\mathrm{OCS}-\mathrm{ME}] \%$} & {$[\mathrm{DC}-\mathrm{ME}] \%$} \\
\hline 44 & 0 & 0,0000 & 0,0000 \\
43 & 1 & - & 0,0113 \\
41 & 3 & - & 0,0341 \\
38 & 6 & 0,0681 & 0,0681 \\
34 & 10 & 0,1140 & 0,1140 \\
30 & 14 & 0,1590 & 0,1590 \\
26 & 18 & 0,2050 & 0,2050 \\
22 & 22 & 0,2500 & 0,2500 \\
18 & 26 & 0,2950 & 0,2950 \\
14 & 30 & 0,3410 & 0,3410 \\
06 & 38 & 0,4320 & 0,4320 \\
0 & 44 & 0,5000 & 0,5000 \\
\hline
\end{tabular}

\section{Medidas de eficiência de inibição à corrosão}

Os ensaios das medidas de eficiência de inibição à corrosão foram realizados em célula de três eletrodos: eletrodo de referência em Ag/ $\mathrm{AgCl}$ (prata/cloreto de prata), eletrodo auxiliar de platina e o eletrodo 
de trabalho em aço carbono AISI-1020 polido até espelhamento. O potenciostato modelo 273A da Par-E\&GG foi utilizado para as medidas de inibição à corrosão. As análises foram realizadas de acordo com procedimentos previamente relatados ${ }^{31}$. Inicialmente ocorre a imersão dos três eletrodos em uma célula de vidro (com capacidade de $\left.8 \mathrm{~cm}^{3}\right)$ contendo a solução padrão de $\mathrm{NaCl}(0,5 \%)$, que caracteriza o meio corrosivo e serve como referência para a comparação das correntes de corrosão. Na seqüência, aplicou-se um potencial de condicionamento do eletrodo em $-1,0 \mathrm{~V}$ por $10 \mathrm{~min}$, tendo sido realizada uma varredura linear de potencial entre os potenciais de equilíbrio -20 e +20 mV, a uma razão de $1 \mathrm{mV} / \mathrm{s}$. Deste modo, obteve-se a resistência à polarização (RP) de acordo com a Equação 1.

$\mathrm{E}=\mathrm{E}_{\text {corr }}+\frac{\mathrm{RT}}{\mathrm{nFi}_{\mathrm{o}}} \mathrm{i}$

onde $\frac{\mathrm{RT}}{\mathrm{nFi}_{0}}$ é a resistência de polarização

Para cada concentração de SME analisado (Tabela 1) a medida de resistência de polarização foi repetida até que o desvio padrão fosse menor que $5 \%$ da média. $\mathrm{O}$ cálculo da eficiência de inibição foi baseado na Equação 2.

$\theta=\left(\mathrm{R}-\mathrm{R}_{0}\right) / \mathrm{R}$

onde $\mathrm{R}_{0}$ é a resistência de polarização para uma solução que não contém inibidor.

\section{RESULTADOS E DISCUSSÃO}

Os sistemas microemulsionados são sistemas interfaciais sofisticados produzidos espontaneamente pela auto-organização de moléculas tensoativas nas interfaces óleo-água, formando microestruturas dispersas em um meio contínuo ${ }^{32}$. Neste trabalho, a escolha do tensoativo OCS se deu em função de ser um produto regional de fácil obtenção, que apresenta uma grande região de microemulsão (ME) do tipo óleo/água $(\mathrm{O} / \mathrm{A})^{25}$. O óleo de coco saponificado (OCS) foi obtido a partir de óleo de coco que foi previamente submetido a análises físico-químicas [índices de acidez $(12,77)$, iodo $(7,66)$ e saponificação $(228,43)]$ de acordo com metodologia previamente relatada ${ }^{30}$. Estas análises são imprescindíveis para a determinação da massa de $\mathrm{NaOH}$ (21,5 g) necessária para a saponificação deste óleo ${ }^{30}$. O critério de escolha do cotensoativo (butanol) está correlacionado com uma maior amplitude da região de $\mathrm{ME}$, tendo sido previamente evidenciado que o butanol é pouco solúvel em óleo e em água e apresenta baixo poder de solubilização frente ao $\mathrm{OCS}^{33}$. Adicionalmente, o comprimento da cadeia do OCS não deve ultrapassar o comprimento da soma das cadeias carbônicas de ambos cotensoativo e óleo (no caso, querosene) $)^{33}$. Desta forma, a penetração do filme interfacial pelas moléculas do óleo $\left(\mathrm{F}_{\mathrm{O}}\right)$ favorece a obtenção de sistema $\mathrm{ME}$ onde a região predominante é O/A (a fase dispersa é o óleo e a contínua, a água). Neste tipo de sistema, a amplitude da $\mathrm{F}_{\mathrm{O}}$, bem como a polaridade, conduz a fortes efeitos de solvatação (tensoativo/óleo) sobre a interface. Portanto, o aumento de ambos, volume molecular do componente da $\mathrm{F}_{\mathrm{O}}$ e polaridade, favorece a redução de interações entre microgotículas do sistema ME obtido, diminuindo o seu poder de solubilização ${ }^{34}$. A razão cotensoativo/tensoativo $(\mathrm{C} / \mathrm{T})$ é um fator fundamental para o aumento do poder de solubilização do sistema ME, no caso (tensoativo OCS) uma razão $\mathrm{C} / \mathrm{T}=1$ forneceu diagramas pseudoternários com três regiões de Winsor (WIV, WIV e WII), onde a região WIV do tipo O/A foi a região de trabalho.

Inicialmente utilizou-se o meio salino em três concentrações $(\mathrm{NaCl}$
0,5, 1,5 e 2,5\%), tendo sido possível comprovar que o aumento da concentração de $\mathrm{NaCl}$ reduz a região de trabalho (Winsor IV), como mostrado na Figura 2. A justificativa para esta redução é dada em função de forças coulombianas das cabeças polares de tensoativos em geral, já que o aumento do teor salino provoca aumento da afinidade do tensoativo com a fase oleosa [no caso, OCS (tensoativo) e querosene $\left(\mathrm{F}_{\mathrm{O}}\right)$ ]. Portanto, a utilização da menor concentração salina $(\mathrm{NaCl} 0,5)$ possibilitou a amplitude da região de microemulsão (Winsor IV). Desta forma, escolheu-se um ponto na região de Winsor IV $\left(30 \% \mathrm{C} / \mathrm{T} ; 60 \% \mathrm{~F}_{\mathrm{A}} ; 10 \% \mathrm{~F}_{\mathrm{O}}\right)$ no diagrama (Figura 2a) que apresentou a seguinte composição: cotensoativo/tensoativo $\mathrm{C} / \mathrm{T}=1(30 \%)$, onde o OCS foi o tensoativo utilizado (15\%), o butanol (15\%) foi o cotensoativo, o querosene foi a fase orgânica $\left(\mathrm{F}_{\mathrm{o}}\right)$ e a solução de $\mathrm{NaCl} 0,5 \%$ foi a fase aquosa $\left(\mathrm{F}_{\mathrm{A}}\right)$.

A análise dos diagramas obtidos (Figura 2) mostrou que o tensoativo OCS possui caráter hidrofílico (a região de trabalho é do tipo O/A, ou seja, rica em água) que pode estar correlacionado com o seu volume molecular (ácido octanóico 7,6\%; ácido decanóico 7,3\%; ácido láurico 48,2\%; ácido mirístico 1,6\%; ácido palmítico 8,0\%; ácido esteárico 3,8\%; ácido oléico 5,0\%; ácido linoléico 2,5\%) ${ }^{25}$ facilitando a formação de micelas diretas, onde a interação interfacial óleo/ água é minimizada pela presença do tensoativo.

\section{Eficiência de inibição à corrosão}

A solubilização da DC no sistema OCS-ME resultou de várias análises, tendo sido possível comprovar a eficácia do sistema na seguinte composição: $30 \%$ de $\mathrm{C} / \mathrm{T}, 60 \%$ de $\mathrm{F}_{\mathrm{A}}$ e $10 \%$ de $\mathrm{F}_{\mathrm{O}}$, onde ocorreu $100 \%$ de solubilização $(0,0126$ g DC em $2 \mathrm{~mL} \mathrm{ME})$ da DC, sendo obtido o sistema DC microemulsionada (DC-ME).

A Figura 3 apresenta as eficiências de inibição à corrosão de OCS puro (3a), OCS-ME (3b) e DC-ME (3c), onde as curvas de eficiência observadas versus concentração destes componentes e suas isotermas de Langmuir e Frumkin foram analisadas comparativamente. De acordo com o esperado, pode-se constatar que: as diferenças observadas entre as moléculas adsorvidas, em cada sistema (Figura 3), indicaram a seguinte ordem de eficiência de inibição à corrosão: OCS com eficiência máxima de $63 \%$ com concentração na faixa de $0,14-0,16 \%$, OCSME com eficiência máxima de $77 \%$ na faixa de com concentração $0,5 \%$ e DC-ME com eficiência máxima de $92 \%$ com concentração na faixa de $0,48-0,50 \%$.

Como pode ser observado na Figura 3, a isoterma de Frumkin que utiliza os parâmetros discriminados na Tabela 2 aproxima-se mais dos dados experimentais que a isoterma de Langmuir. $\mathrm{Na}$ isoterma de Frumkin as constantes de adsorção $(\mathrm{K})$ e os parâmetros de interação lateral (A) de OCS, OCS-ME e DC-ME apresentaram melhores resultados. Estes parâmetros foram determinados utilizando-se a minimização direta a partir da Equação 3, com um software em QBASIC $^{\circledR}$ do método de otimização simplex Nelder-Mead ${ }^{35}$. Este método consiste em determinar os valores dos parâmetros da isoterma a partir da eficiência de inibição à corrosão medida versus a eficiência calculada (Equação 3). No caso, esta eficiência foi obtida para os inibidores OCS, OCS-ME e DC-ME, tendo sido minimizados os melhores pontos de $\mathrm{K}$ e A.

$\mathrm{F}(\mathrm{K}, \mathrm{A})=\sum_{\mathrm{i}=1}^{\mathrm{N}}\left(\frac{\theta_{\mathrm{cal}, \mathrm{i}^{-}{ }_{\mathrm{obs}, \mathrm{i}}}}{\theta_{\mathrm{obs}, \mathrm{i}}}\right)^{2}$

A constante de adsorção de OCS-ME na isoterma de Frumkin $(3,0)$ é menor que a constante de adsorção de OCS puro (203), devido à maior solubilização do OCS no sistema ME. Apesar de OCS-ME apresentar uma adsorção tipicamente física (os efeitos entre o metal e o inibidor OCS são relativamente fracos), suas mo- 


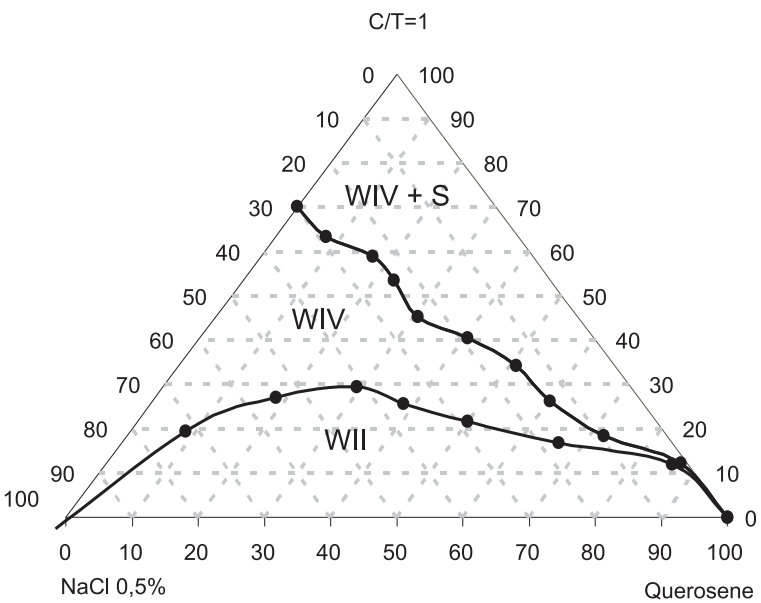

(a) $0,5 \%$ de $\mathrm{NaCl}$

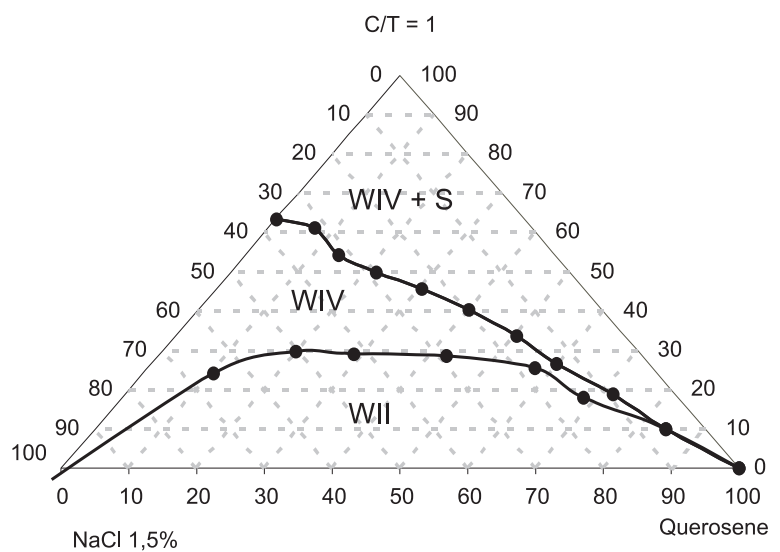

(b) $1,5 \%$ de $\mathrm{NaCl}$

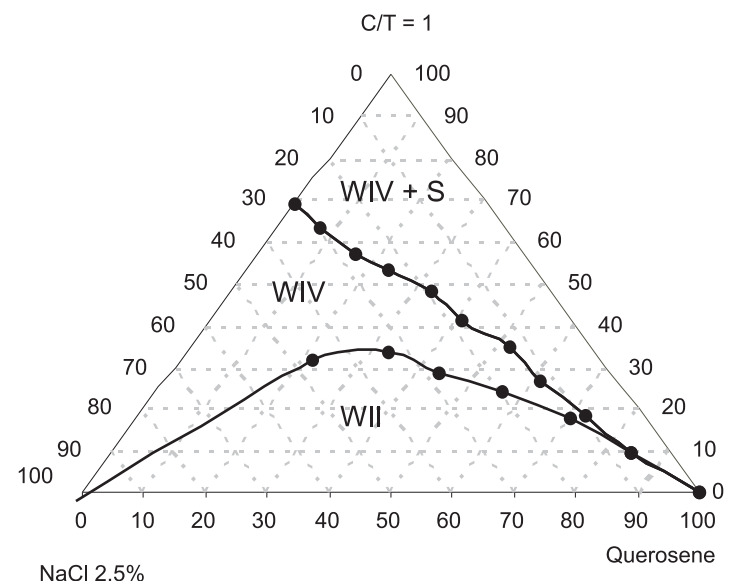

(c) $2,5 \%$ de $\mathrm{NaCl}$

Figura 2. Composições para o diagrama de fases: tensoativo (OCS), cotensoativo (butanol), fase aquosa (solução de $\mathrm{NaCl}$ ) e fase orgânica (querosene)

léculas podem criar uma barreira de proteção do eletrodo, apontada pela interação lateral atrativa que existe entre elas.

$\mathrm{O}$ valor elevado (780) do K da DC-ME indica maior adsorção desta substância na interface, possivelmente devido à presença dos quatro grupos aminas e do grupo carbonila. A presença destes grupos promove uma maior interação da molécula com a superfície metálica,

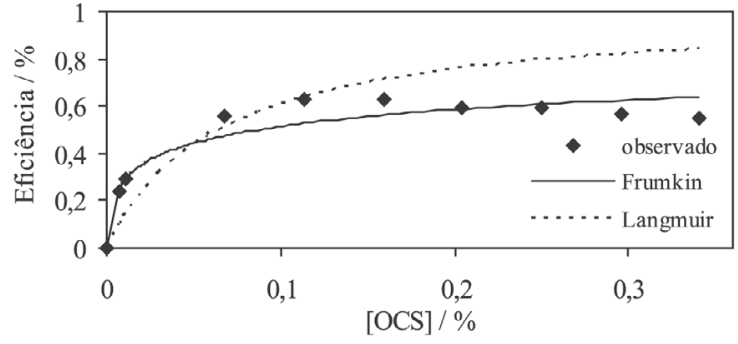

a) OCS puro

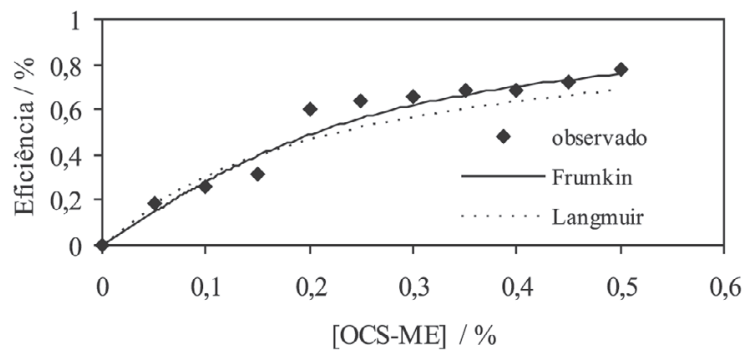

b) OCS-ME

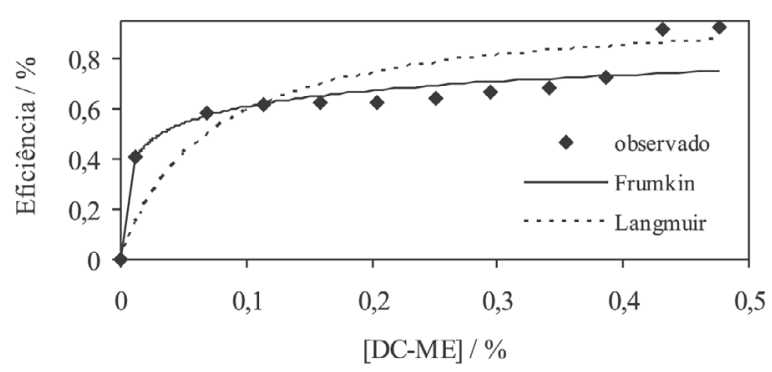

c) $\mathrm{DC}-\mathrm{ME}$

Figura 3. Eficiência de inibiação à corrosão de OCS puro, OCS-ME e DC$M E$

Tabela 2. Constantes de adsorção e parâmetros de interação lateral para os inibidores de corrosão OCS puro, OCS-ME e DC-ME

\begin{tabular}{lcccccc}
\hline & \multicolumn{2}{c}{ OCS puro } & \multicolumn{2}{c}{ OCS-ME } & \multicolumn{2}{c}{ DC-ME } \\
& $\mathrm{K}$ & $\mathrm{A}$ & $\mathrm{K}$ & $\mathrm{A}$ & $\mathrm{K}$ & $\mathrm{A}$ \\
\hline Langmuir & 15,4 & -- & 4,4 & -- & 14,1 & -- \\
Frumkin & 203 & 5,74 & 3,0 & $-1,0$ & 780 & 6,5 \\
\hline
\end{tabular}

favorecendo a inibição à corrosão. Desta forma, o poder anticorrosivo do sistema DC-ME foi mais eficaz que o sistema OCS-ME.

De acordo com o parâmetro de interação lateral de DC-ME $(6,5)$ que suplantou o parâmetro de $\operatorname{OCS}-\mathrm{ME}(-1,0)$, a interação repulsiva entre as moléculas no sistema DC-ME deve ser significativamente grande. Possivelmente, o tamanho dos grupos aromáticos presentes na molécula da DC favoreça interações repulsivas entre as moléculas do tensoativo (OCS) e as moléculas da DC, repercutindo no aumento do valor da interação lateral de DC-ME. Apesar desta forte interação lateral repulsiva, os valores dos parâmetros A e K obtidos para OCS e DC-ME são comparativamente compatíveis, o que indica que as interações OCS/metal e DC-ME/metal são comparáveis, isto é, interagem fortemente com o eletrodo. Por outro lado, o tensoativo microemulsionado OCS$\mathrm{ME}$, apesar de ter apresentado interação mais fraca com o eletrodo, interagiu lateralmente de modo atrativo. 


\section{CONCLUSÕES}

O sistema de microemulsão obtido com óleo de coco saponificado (OCS-ME) mostrou elevado poder de solubilização da substância testada (DC). Os inibidores de corrosão testados OCS (solubilizado em água), OCS-ME (OCS-microemulsionado) e DC-ME (difenilcarbazida-microemulsionada) foram bastante eficientes, sendo verificado que mesmo em baixas concentrações, são eficazes, já que o OCS puro apresentou 63\% de inibição à corrosão, OCS-ME $77 \%$ e a DC-ME $92 \%$. Desta forma, comprovou-se que o sistema microemulsionado ampliou o poder anticorrosivo de OCS, já que a eficiência máxima de inibição de OCS-ME sofreu um acréscimo significativo (14\%). Este fenômeno pode ser justificado pela adsorção do tensoativo (OCS) na interface líquido-sólido formando uma camada protetora mais homogênea sobre o metal, o que possibilita maior contato interfacial devido à área apresentada pelas microestruturas formadas.

O fato da DC não ser solúvel em meio neutro impossibilitou a realização de testes com a substância pura, dificultando esclarecimentos sobre a possibilidade da elevada eficiência observada para DC-ME (92\%) estar correlacionada com o SME ou ainda, com um possível efeito sinérgico entre OCS e DC. No entanto, estudos anteriores mostraram que a DC solubilizada em meios ácido e básico apresentou eficácia máxima de inibição à corrosão em torno de $85 \%{ }^{9-12}$. Adicionalmente, sabe-se que sistemas ricos em oxigênio, nitrogênio e anel aromático (apresentam elevada distribuição de cargas) são inibidores eficientes ${ }^{4}$. Neste contexto, o fato da DC possuir características estruturais que favoreçam sua utilização como inibidor de corrosão, bem como ter sido solúvel em um sistema microemulsionado, influenciaram significantemente no seu poder anticorrosivo.

\section{REFERÊNCIAS}

1. Gentil, V.; Corrosão, $3^{\mathrm{a}}$ ed., Livros Técnicos e Científicos S. A.: Rio de Janeiro, 1996.

2. Shreir, L. L.; Jarman, R. A.; Burstein, G. T.; Corrosion: Metal/Environment Reactions, Butterworth-Heinemann: London, 2000, p. 38.

3. Clint, J. H.; Surfactant aggregation, Blackie \& Son Ltd.: Glasgow, 1992.

4. Ebenso, E. E.; Ekpe, U. J.; Ita, B. I.; Offiong, O. E.; Ibok, U. J.; Mater. Chem. Phys. 1999, 60, 79.

5. El-Achouri, M.; Infante, M. R.; Izquierdo, F.; Kertit, S.; Gouttaya, H. M.; Nciri, B.; Corros. Sci. 2001, 43, 19.
6. Alsabagh, A. M.; Migahed, M. A.; Hayam, S. A.; Corros. Sci. 2006, 48, 813.

7. Ghasemi, Z.; Tizpar, A.; Appl. Surf. Sci. 2006, 252, 3667.

8. Abdel Hamid, Z.; Soror, T. Y.; El-Dahan, H. A.; Omar, A. M. A.; Anticorros. Methods \& Mater. 1998, 45, 306.

9. Patel, N. K.; Franco, J.; Patel, L. S.; Chemical Era 1975, 11, 20.

10. Ismail, A. A.; Sandad, S. H.; El-Meligi, A. A.; Bull. Electrochem. 1994, $10,448$.

11. Fouda, A. S.; Madkour, L. H.; El-Shafei, A. A.; Eimaksoud, S. A.; Bull. Korean Chem. Soc. 1995, 16, 454.

12. Sandad, S. H.; Ismail, A. A.; El-Meligi, A. A.; Bull. Electrochem. 1995, 11,462 .

13. Marckwald, W.; Chemistry Ber 1942, 25, 3098.

14. Yale, H. L.; Losee, K. A.; Perry, F. M.; J. Am. Chem. Soc. 1954, 76, 2208.

15. El-Achouri, M.; Hajji, M. S.; Kertit, S.; Essassi, E. M.; Salem, M.; Coudert, R.; Corros. Sci. 1995, 37, 381.

16. El-Achouri, M.; Hajji, M. S.; Salem, M.; Kertit, S.; Aride, J.; Coudert, R.; Essassi, E. M.; Corros. Sci. 1996, 52, 103.

17. Li, X.; Mu, G.; Appl. Surf. Sci. 2005, 252, 1254.

18. Schmitt, G.; Br. Corrosion J. 1984, 19, 165.

19. Frignani, A.; Zucchi, F.; Monticelli, C.; Br. Corrosion J. 1983, 18, 19.

20. Branzoi, V.; Branzoi, F.; Baibarac, M.; Mater. Chem. Phys. 2000, 65, 288.

21. Banerjje, G.; Malhotra, S. N.; Corrosion 1992, 48, 10.

22. Castro Dantas, T. N.; Ferreira Moura, E.; Scatena Jr, H.; Dantas Neto, A. A.; Corrosion 2002, 58, 723.

23. Castro Dantas, T. N.; Dantas Neto, A. A.; Moura, M. C. P. A.; Water Res. 2001, 35, 2219.

24. Castro Dantas, T. N.; Dantas Neto, A. A.; Moura, M. C. P. A.; Barros Neto, E. L.; Paiva Telêmaco, E.; Langmuir 2001, 17, 4256.

25. Silva, A. C. M.; Castro Dantas, T. N.; Barros Neto, E. L.; Ind. Eng. Chem. Res. 2002, 41, 3042.

26. Castro Dantas, T. N.; Dantas Neto, A. A.; Moura, M. C. P. A.; Barros Neto, E. L.; Forte, K. R.; Leite, R. H. L.; Water Res. 2003, 37, 2709.

27. Chen, H.; Chang, X.; Weng, T.; Zhao, X.; Gao, Z.; Yang, Y.; Xu, H.; Yang, X.; J. Controlled Release 2004, 98, 427.

28. Oliveira, A. G.; Scarpa, M. V.; Correa, M. A.; Cera, L. F. R.; Formariz, T. P.; Quim. Nova 2004, 27, 131.

29. Castro Dantas, T. N.; Lucena Neto, M. H.; Dantas Neto, A. A.; Moura, M. C. P. A.; Barros Neto, E. L.; Ind. Eng. Chem. Res. 2005, 44, 6784

30. Moreto, E.; Fett, R.; Óleos e Gorduras Vegetais: Processamento e Análises, Ed. da UFSC: Florianópolis, 1989, p. 126, 141, 144.

31. Wolynec, S.; Técnicas Eletroquímicas em Corrosão, Ed. da USP: São Paulo, 2003.

32. Atwood, D.; Florence, A. T.; Surfactants Systems: Their Chemistry, Pharmacy and Biology, Chapman and Hall: London, 1985.

33. Leite, R. H. L.; Tese de Mestrado, Universidade Federal do Rio Grande do Norte, Brasil, 1995.

34. Friberg, S. R.; Venable, R. L.; Encyclopedia of emulsion technology, Ed. P. Becher: New York, 1983.

35. Prosser, A. J.; Frances, E. I.; Colloids Surf., A 2001, 178, 1. 